\title{
Introduction to Nanoscale Thermal Conduction
}

\author{
Patrick E. Hopkins ${ }^{1,2}$ and John C. Duda ${ }^{2}$ \\ ${ }^{1}$ Sandia National Laboratories, Albuquerque, New Mexico \\ ${ }^{2}$ University of Virginia, Charlottesville, Virginia \\ United States of America
}

\section{Introduction}

Thermal conduction in solids is governed by the well-established, phenomenological Fourier Law, which in one-dimension is expressed as

$$
Q=-\kappa \frac{\partial T}{\partial z}
$$

where the thermal flux, $Q$, is related to the change in temperature, $T$, along the direction of thermal propagation, $z$, through the thermal conductivity, $\kappa$. The thermal conductivity is a temperature dependent material property that is unique to any given material. Figure 1 shows the measured thermal conductivity of two metals - $\mathrm{Au}$ and $\mathrm{Pt}$ - and two semiconductors - Si and Ge (Ho et al., 1972). In these bulk materials, the thermal conductivities span three orders of magnitude over the temperature range from $1-1,000 \mathrm{~K}$. Temperature trends in the thermal conductivities are similar depending on the type of material, i.e., Si and Ge show similar thermal conductivity trends with temperature as do Au and Pt. These similarities arise due to the different thermal energy carriers in the different classes of materials.

In metals, heat is primarily carried by electrons, whereas in semiconductors, heat moves via atomic vibrations of the crystalline lattice. The macroscopic average of these carriers' scattering events, which is related to the thermal conductivity of the material, gives rise to the spatial temperature gradient in Eq. 1. This temperature gradient is established from the energy carriers traversing a certain distance, the mean free path, before scattering and losing their thermal energy. In bulk systems, this mean free path is related to the intrinsic properties of the materials. However, in material systems with engineered length scales on the order of the mean free path, additional scattering events arise due to energy carrier scattering with interfaces, inclusions, grain boundaries, etc. These scattering events can substantially change the thermal conductivity of nanostructured systems as compared to that of the bulk constituents (Cahill et al., 2003). In fact, in any given material in which the physical size is less than the mean free path, the carrier scattering events will only occur at the boundaries of the material. Therefore, there will be no temperature gradient established in the material and Eq. 1 will no longer be valid.

Typical carrier room temperature mean free paths in metals and semiconductors are about 10 $100 \mathrm{~nm}$, respectively (Tien et al., 1998). Clearly, with the wealth of technology and applications that rely on material systems with characteristic lengths scales in the sub- $1.0 \mu \mathrm{m}$ regime (Wolf, 2006), the need to understand thermal conduction at the nanoscale is immensely important for thermal management and engineering applications. In this chapter, the basic concepts of 
nanoscale thermal conduction are introduced at the length scales of the fundamental energy carriers. This discussion will begin by introducing the kinetic theory of gases, specifically mean free path and scattering time, and how these concepts apply to thermal conductivity. Then, the properties of solids will be discussed and the concepts of lattice vibrations and density of states will be quantified. The link from this microscopic, individual energy carrier development to bulk properties will come with the discussion of statistical mechanics of the energy carriers. Finally, transport properties will be discussed by quantifying carrier scattering times in solids, which will lead to the derivation of the thermal conductivity from the individual energy carrier development in this chapter. In the final section, this expression for thermal conductivity will be used to model the thermal conductivity of nanosystems by accounting for energy carrier scattering times competing with boundary scattering effects.

\section{Kinetic theory}

Heat transfer involves the motion of particles, quasi-particles, or waves generated by temperature differences. Given the position and velocity of any of these energy carriers, their motion determines the heat transfer. If energy carriers are treated as particles, as will be the focus of this chapter, then the heat transfer can be analyzed through the Kinetic Theory of Gases (Vincenti \& Kruger, 2002). For a discussion of nanoscale thermal conduction by waves, see Chapter 5 of Chen (2005).

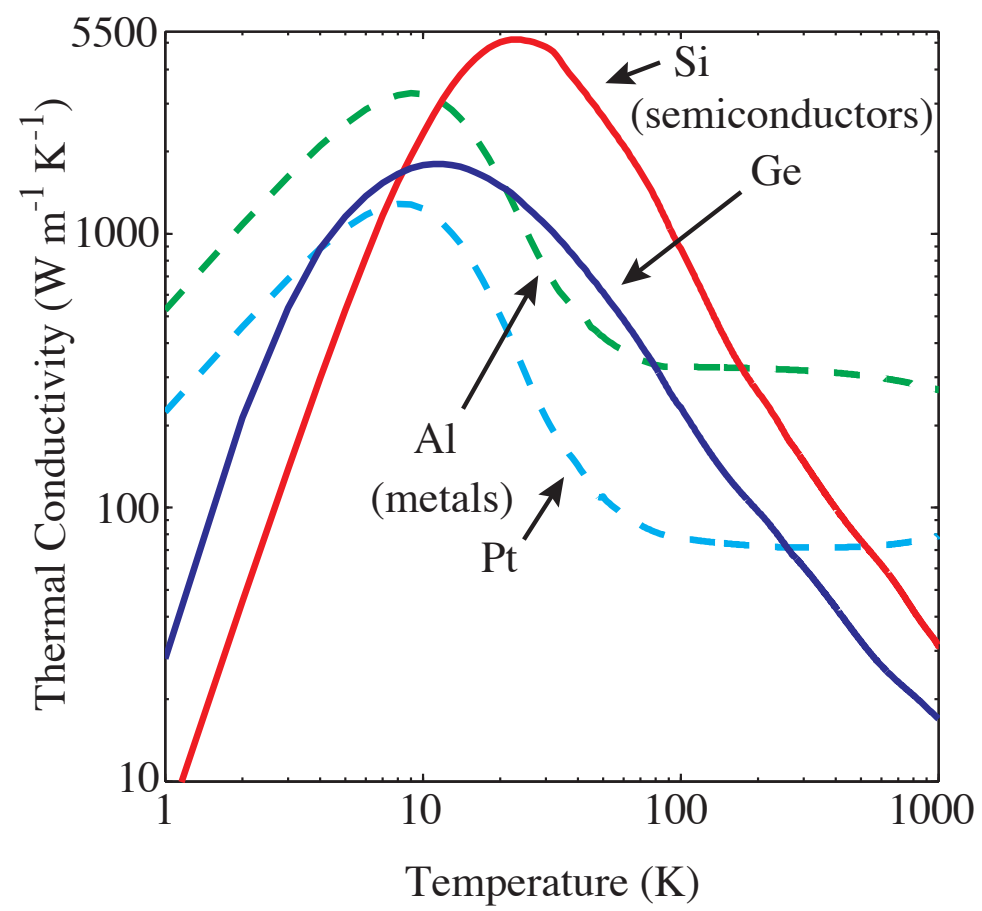

Fig. 1. Measured thermal conductivity of two metals (aluminum and platinum) and two semiconductors (silicon and germanium) (Ho et al., 1972). 
Consider a one-dimensional flow of energy across an imaginary surface perpendicular to the energy flow direction. The net heat flux across this surface is the difference between the thermal fluxes of the carriers flowing in the positive and negative directions, $Q_{+z}$ and $Q_{-z}$ respectively. The carriers with energy $\varepsilon$ will travel a distance before experiencing a scattering event that causes the carriers to change direction and/or transfer energy; this distance is called the mean free path, $\lambda=v_{z} \tau$, where $v_{z}$ is the particle velocity in the $z$-direction (direction of heat flow) and $\tau$ is the relaxation time, or the average time a heat carrier travels before it is scattered and changes direction and/or transfers energy. Therefore, given a volumetric carrier number density, $n$, the net heat flux in the $\mathrm{z}$-direction is

$$
Q_{z}=Q_{+z}+Q_{-z}=\frac{1}{2}\left(\left.\left(n \epsilon v_{z}\right)\right|_{v_{z+\lambda}}+\left.\left(-n \epsilon v_{z}\right)\right|_{v_{z-\lambda}}\right)
$$

which can be re-expressed as

$$
Q_{z}=-v_{z} \tau \frac{\partial\left(n \epsilon v_{z}\right)}{\partial z}
$$

Now, given an isotropic medium, the average velocity is $v_{z}^{2}=v^{2} / 3$, and thus, the average flux is given by

$$
Q_{z}=-\frac{v^{2}}{3} \tau \frac{\partial(n \epsilon)}{\partial z}
$$

Defining a volumetric energy density, or internal energy, as $U=n \epsilon$, and applying the chain rule to the derivative in $z$ yields

$$
Q_{z}=-\frac{v^{2}}{3} \tau \frac{d U}{d T} \frac{d T}{d z}
$$

The temperature derivative of the internal energy is defined as the volumetric heat capacity, $C=d U / d T$, and with this, comparing Eq. 5 with Eq. 1 yields

$$
\kappa=\frac{1}{3} C v^{2} \tau=\frac{1}{3} C v \lambda
$$

Equation 6 defines the thermal conductivity of a material based on the properties of the energy carriers in the solid. To calculate the thermal conductivity of the individual energy carriers in a solid, and therefore understand how $\kappa$ changes on the nanoscale, the volumetric heat capacity, carrier velocity, and scattering times must be known. This will be the focus of the remainder of this chapter.

\section{Energy states}

The allowed energies of thermal carriers in solids are dictated by the periodicity of the atoms that comprise the solid. Atoms in a crystal are arranged in a basic primitive cell that is repeated throughout the crystalline volume. The atoms that comprise the primitive cell are called the basis of the crystal, and the arrangement in which this basis is repeated is called the lattice. As a full treatment of the solid state crystallography is beyond the scope of this chapter, the reader is directed to more information on crystallography and solid lattices in any introductory solid state physics (Kittel, 2005) or crystallography text (Ziman, 1972). However, the important thing to remember is that, for the development in this section, periodicity in the atomic arrangement gives rise to the available energy states in a solid.

At this point, the discussion is focused on the primary thermal energy carriers in a solid, i.e., electrons and lattice vibrations (phonons). In the subsections that follow, the fundamental 
equations governing the motion of electron and lattice waves through a one-dimensional crystalline lattice will be introduced, and then the effects of periodicity will be discussed. This will give to rise the allowed energy states of the electrons and phonons, which are fundamental to determining $\kappa$.

\subsection{Electrons}

The starting point for describing the allowed motion of electrons through a solid is given by the Schrödinger Equation (Schrödinger, 1926)

$$
-\frac{\hbar^{2}}{2 m} \frac{\partial^{2} \Psi}{\partial z^{2}}+V \Phi=i \hbar \frac{\partial \Psi}{\partial t},
$$

where $\hbar$ is Planck's constant divided by $2 \pi$ (Planck's constant is $h=6.6262 \times 10^{-34} \mathrm{~J} \mathrm{~s}$ ), $m$ is the mass of the electron, $\Psi$ is the electron wavefunction which is dependent on time and space, $V$ is the potential that is acting on the electron system, and $t$ is the time. Equation 7 is the fundamental equation governing the field of quantum mechanics, and only a basic discussion of this equation will be provided in this chapter in order to understand introductory nanoscale thermal conduction. To delve more into this equation, the reader is encouraged to read any text on introductory quantum mechanics (Griffiths, 2000).

For the solid systems of interest in this chapter, the potential $V$ is the interatomic potential, which is related to the force between the atoms in a crystal (or the "glue" that holds the lattice in a periodic arrangement), and can be assumed as independent of time. With this in mind, separation of variables can be performed on Eq. 7 to determine a spatial and temporal solution. The starting point of this is to assume that the wavefunction can be separated into independent spatial and temporal components (which, again, is valid since $V$ is assumed as independent of time), $\Psi(z, t)=\psi(z) \phi(t)=\psi \phi$, where the functionality of the spatial and temporal solutions are dropped for convenience. Substituting this solution into Eq. 7 yields

$$
\left[-\frac{\hbar^{2}}{2 m} \frac{\partial^{2} \psi}{\partial z^{2}}+V \psi\right] \frac{1}{\psi}=i \hbar \frac{\partial \phi}{\partial t} \frac{1}{\phi}=\epsilon,
$$

where $\epsilon$ is a constant eigenvalue solution to Eq. 8. Equation 8 can be immediately solved for $\phi$ yielding

$$
\phi \propto \exp \left[-i \frac{\epsilon}{\hbar} t\right]
$$

It is interesting to note that the form of the expression describing a classical plane wave oscillating in time is given by $\exp [-i \omega t]$, where $\omega$ is the angular frequency of the wave defined as $\omega=2 \pi f$, where $f$ is the frequency of oscillation of the wave, which is of the same form as Eq. 9. From inspection, the Eigenvalues of the electron waves are $\epsilon \propto \hbar \omega$, i.e., the Eigenvalues are the electron energy states. The governing equation for the spatial component, which is called the time-independent Schrödinger Equation, is given by

$$
-\frac{\hbar^{2}}{2 m} \frac{\partial^{2} \psi}{\partial z^{2}}+(V-\epsilon) \psi=0
$$

Given that the solution to Eq. 7 is the solution to the steady state portion multiplied by Eq. 9, the solution of Eq. 10 contains all the pertinent information about the electronic energy states in a periodic crystal. 
To understand the effects of a periodic interatomic potential acting on the electron waves, consider a simple, yet effective, model for the potential experienced by the electrons in a periodic lattice. This model, the Kronig-Penny Model, assumes there is one electron inside a square, periodic potential with a period distance equal to the interatomic distance, $a$, mathematically expressed as

$$
V=\left\{\begin{array}{ccc}
0 & \text { for } & 0<z \leq b \\
V_{0} & \text { for } & -c \leq z \leq 0
\end{array},\right.
$$

subjected to the periodicity requirement given by $V(z+b+c)=V(z)$, where $a=b+c$. Solutions of Eq. 10 subjected to Eq. 11 are

$$
\psi=\left\{\begin{array}{cl}
D_{1} \exp [i M z]+D_{2} \exp [-i M z] & \text { for } \quad 0<z \leq b \\
D_{3} \exp [i L z]+D_{4} \exp [-i L z] & \text { for } \quad-c \leq z \leq 0
\end{array},\right.
$$

where $D_{1}, D_{2}, D_{3}$, and $D_{4}$ are constants determined from boundary conditions,

$$
\epsilon=\frac{\hbar^{2} M^{2}}{2 m}
$$

and

$$
V-\epsilon=\frac{\hbar^{2} L^{2}}{2 m}
$$

with $M$ and $L$ related to the electron energy.

Although the full mathematical derivation of the predicted allowed electron energies will not be pursued here (see, for example, Griffiths (2000)), one important part of this formalism is recognizing that the periodicity in the lattice gives rise to a periodic boundary condition of the wavefunction, given by

$$
\psi(z+(b+c))=\psi(z) \exp [i z(b+c)]=\psi(z) \exp [i k a],
$$

where $k$ is called the wavevector. Equation 15 is an example of the Bloch Theorem. The wavevector is defined by the periodicity of the potential (i.e., the lattice), and therefore, the goal is to determine the allowed energies defined in Eq. 13 as a function of the wavevector. The relationship between energy and wavevector, $\epsilon(k)$, known as the dispersion relation, is the fundamental relationship needed to determine all thermal properties of interest in nanoscale thermal conduction.

After incorporating the Bloch Theorem and continuity equations for boundary conditions of Eq. 12 and making certain simplifying assumptions (Chen, 2005), the following dispersion relation is derived for an electron subjected to a periodic potential in a one-dimensional lattice:

$$
\frac{A}{K} \sin [M c]+\cos [M c]=\cos [k c] .
$$

Here, $A$ is related to the electron energy and atomic potential $V$, and from Eq. 13

$$
M=\sqrt{\frac{2 m \epsilon}{\hbar^{2}}},
$$

such that Eq. 16 becomes

$$
A \sqrt{\frac{\hbar^{2}}{2 m \epsilon}} \sin \left[\sqrt{\frac{2 m \epsilon}{\hbar^{2}}} c\right]+\cos \left[\sqrt{\frac{2 m \epsilon}{\hbar^{2}}} c\right]=\cos [k c] .
$$


Note that the right hand side of Eq. 18 restricts the solutions of the left hand side to only exist between -1 and 1. However, the left hand side of Eq. 18 is a continuous function that does in fact exist outside of this range. An energy-wavevector combination that results in the left hand side of Eq. 18 to evaluating to a number outside of the range from $[-1,1]$ means that an electron cannot exist for that energy-wavevector combination, indicating that electrons can only exist at very specific energies related to the interatomic potential between the atoms in the crystalline lattice. In addition, there is periodicity in the solution to Eq. 18 that arises on an interval of $k=2 \pi / c$. If the interatomic potential is symmetric, then $b=c=2 a$, and the periodicity arises on a length scale of $k=\pi / a$ and is symmetric about $k=0$. This length of periodicity is called a Brillouin Zone and, in a symmetric case as discussed here, only the first Brillouin Zone from $k=0$ to $k=\pi / a$ need be considered due to symmetry and periodicity.

To simplify this picture, now consider the case where the electrons do not "see" the crystalline lattice, i.e., the electrons can be considered free from the interatomic potential. In this case, the electrons are called free electrons. For free electrons, Eqs. 13 and 14 are identical $(L=M)$ and $A=0$, thus Eq. 18 becomes

$$
\cos \left[\sqrt{\frac{2 m \epsilon}{\hbar^{2}}} c\right]=\cos [k c] .
$$

From inspection, the free electron dispersion relation is given by

$$
\epsilon=\frac{\hbar^{2} k^{2}}{2 m}
$$

This approach of deriving the free electron dispersion relation given by Eq. 20 is a bit involved, as the Schrödinger Equation was solved for some periodic potential, and the result was simplified to the free electron case by assuming the electrons did not "feel" any of the interatomic potential (i.e., $V=0$ ). A bit more straightforward way of finding this free electron dispersion relation is to solve the Schrödinger Equation assuming $V=0$. In this case, the time-independent version of the Schrödinger Equation (Eq. 10) is given by

$$
-\frac{\hbar^{2}}{2 m} \frac{\partial^{2} \psi}{\partial z^{2}}-\epsilon \psi=0
$$

This ordinary differential equation is easily solvable. Rearranging Eq. 21 yields

$$
\frac{\partial^{2} \psi}{\partial z^{2}}+\frac{2 m \epsilon}{\hbar^{2}} \psi=0
$$

The solution to the above equation takes the form

$$
\psi=D_{5} \exp \left[-i \sqrt{\frac{2 m \epsilon}{\hbar^{2}} z}\right]+D_{6} \exp \left[i \sqrt{\frac{2 m \epsilon}{\hbar^{2}} z}\right]
$$

where the wavevector of this plane wave solution is given by $k=\sqrt{2 m \epsilon / \hbar^{2}}$, which yields the same dispersion relationship as given in Eq. 20. Note that the dispersion relationship for a free electron is parabolic $\left(\epsilon \propto k^{2}\right)$. For every $k$ in the dispersion relation, there are two electrons of the same energy with different spins. Although this is not discussed in detail in this development, it is important to realize that since two electrons can occupy the same energy at a given wavevector $k$ (albeit with different spins), the electron energies are considered degenerate, or more specifically, doubly degenerate. 
Although the mathematical development in this work focused on the free electron dispersion, it is important to note the role that the interatomic potential will have on the dispersion. Following the discussion below Eq. 18, the potential does not allow certain energy-wavevector combinations to exist. This manifests itself at the Brillouin zone edge and center as a discontinuity in the dispersion relation. This discontinuity is called a band gap. In practice, for electrons in a single band, the dispersion is often approximated by the free electron dispersion, since only at the zone center and edge does the electron dispersion feel the effect of the interatomic potential. This is a important consideration to remember in the discussion in Section 4

Where the dispersion gives the allowed electronic energy states as a function of wavevector, how the electrons fill the states defines the material as either a metal or a semiconductor. At zero temperature, the filling rule for the electrons is that they always fill the lowest energy level first. Depending on the number of electrons in a given material, the electrons will fill up to some maximum energy level. This topmost energy level that is filled with electrons at zero Kelvin is called the Fermi level. Therefore, at zero temperature, all states with energies less than the Fermi energy are filled and all states with energies greater than the Fermi energy are empty. The location of the Fermi energy dictates whether the material is a metal or a semiconductor. In a metal, the Fermi energy lies in the middle of a band. Therefore, electrons are directly next to empty states in the same band and can freely flow throughout the crystal. This is why metals typically have a very high electrical conductivity. For this reason, the majority of the thermal energy in a metal is carried via free electrons. In a semiconductor, the Fermi energy lies in the middle of the band gap. Therefore, electrons in the band directly below the Fermi energy are not adjacent to any empty states and cannot flow freely. In order for electrons to freely flow, energy must be imparted into the semiconductor to case an electron to jump across the band gap into the higher energy band with all the empty states. This lack of free flowing electrons is the reason why semiconductors have intrinsically low electrical conductivity. For this reason, electrons are not the primary thermal carrier in semiconductors. In semiconductors, heat is carried by quantized vibrations of the crystalline lattice, or phonons.

\subsection{Phonons}

A phonon is formally defined as a quantized lattice vibration (elastic waves that can exist only at discrete energies). As will become evident in the following sections, it is often convenient to turn to the wave nature of phonons to first describe their available energy states, i.e., the phonon dispersion relationship, and later turn to the particle nature of phonons to describe their propagation through a crystal.

In order to derive the phonon dispersion relationship, first consider the equation(s) of motion of any given atom in a crystal. To simplify the derivation without losing generality, attention is given to the monatomic one-dimensional chain illustrated in Fig. 2a, where $m$ is the mass of the atom $j, K$ is the force constant between atoms, and $a_{1}$ is the lattice spacing. The displacement of atom $m_{j}$ from its equilibrium position is given by,

$$
u_{j}=x_{j}-x_{j}^{o},
$$

where $x_{j}$ is the displaced position of the atom, and $x_{j}^{o}$ is the equilibrium position of the atom. Likewise, considering similar displacements of nearest neighbor atoms along the chain and 
applying Newtown's law, the net force on atom $m_{j}$ is

$$
F_{j}=K\left(u_{j+1}-u_{j}\right)+K\left(u_{j-1}-u_{j}\right) .
$$

Collecting like terms, the equation of motion of atom $m_{j}$ becomes

$$
m \ddot{u}_{j}=K\left(u_{j+1}-2 u_{j}+u_{j-1}\right),
$$

where $\ddot{u}_{j}$ refers to the double derivative of $u_{j}$ with respect to time. It is assumed that wavelike solutions satisfy this differential equation and are of the form

$$
u_{j} \propto \exp \left[i\left(k a_{1}-\omega t\right)\right]
$$

where $k$ is the wavevector. Substituting Eq. 27 into Eq. 26 and noting the identity $\cos x=$ $2\left(e^{i x}+e^{-i x}\right)$ yields the expression

$$
m \omega^{2}=2 K\left(1-\cos \left[k a_{1}\right]\right) .
$$

Finally, the dispersion relationship for a one-dimensional monatomic chain can be established by solving for $\omega$,

$$
\omega(k)=2 \sqrt{\frac{K}{m}}\left|\sin \left[\frac{1}{2} k a_{1}\right]\right| .
$$

Just as was the case with electrons, attention is paid only to the solutions of Eq. 29 for $-\pi / a_{1} \leq k \leq \pi / a_{1}$, i.e., within the boundaries of the first Brillouin zone. A plot of the dispersion relationship for a one-dimensional monatomic chain is shown in Fig. 3a. It is important to notice that the solution of Eq. 29 does not change if $k=k+2 \pi N / a_{1}$, where

(a)

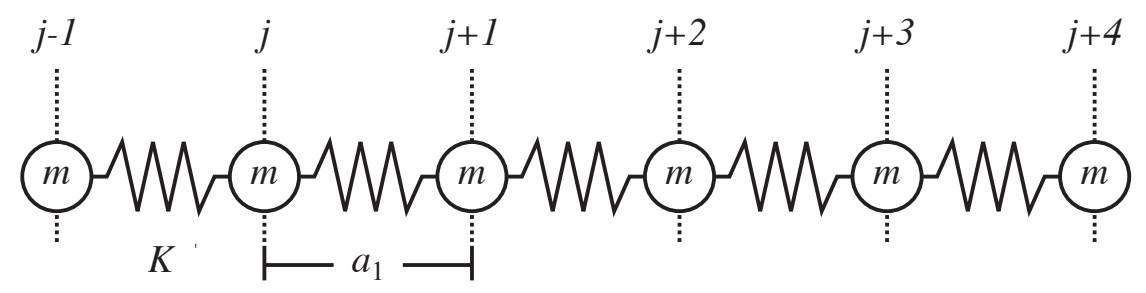

(b)

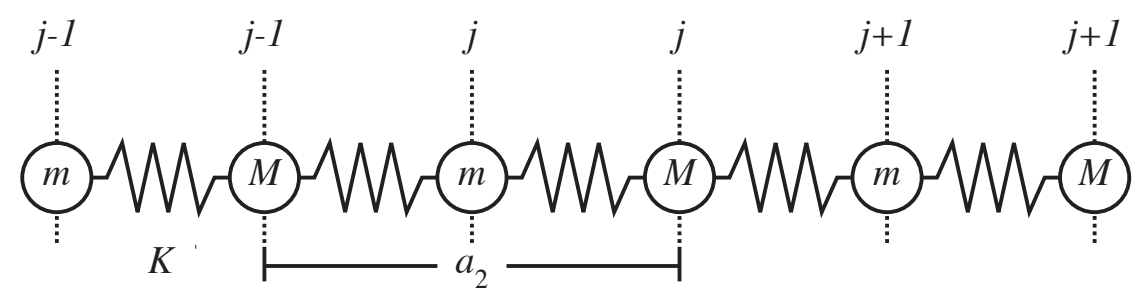

Fig. 2. Schematics representing (a) monatomic and (b) diatomic one-dimensional chains. Here, $m$ and $M$ are the masses of type-A and type-B atoms, $a_{1}$ and $a_{2}$ are the respective lattice constants of the monatomic and diatomic chains, and $K$ is the interatomic force constant. 

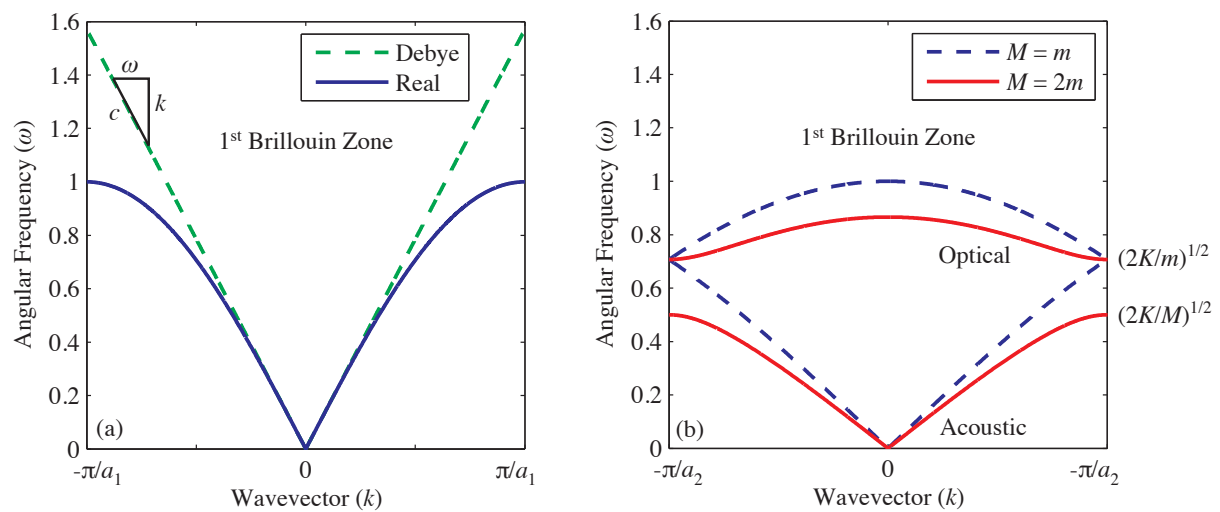

Fig. 3. (a) Phonon dispersion relationship of a one-dimensional monatomic chain as presented in Eq. 29. Also plotted is the corresponding Debye approximation. Note that not only does the Debye approximation over-predict the frequency of phonons near the zone edge, but it also predicts a non-zero slope, and thus, a non-zero phonon group velocity at the zone edge. (b) Phonon dispersion relationship of a one-dimensional diatomic chain as presented in Eq. 35. In the case where $M=m$, the dispersion is identical to that plotted in (a), but is represented in a "zone folded" scheme. The size of the phononic band gap depends directly on the difference between the atoms comprising the diatomic chain.

$N$ is an integer. This indicates that all vibrational information is contained within the first Brillouin zone.

A phonon dispersion diagram concisely describes two essential pieces of information required to describe the propagation of lattice energy in a crystal. First, as is obvious from Eq. 29, the energy of a given phonon, $\hbar \omega$, is mapped to a distinct wavevector, $k$ (in turn, this wavevector can be related to the phonon wavelength). As might be expected, longer wavelength phonons are associated with lower energies. Second, the group velocity, or speed at which a "packet" of phonons propagates, is described by the relationship

$$
v_{g}=\frac{\partial \omega}{\partial k},
$$

where $v_{g}$ is the phonon group velocity. Additional insight can be gained if focus is turned to two particular areas of the dispersion relationship: the zone center $(k=0)$ and the zone edge $\left(k=\pi / a_{1}\right)$.

Discussion of phonons at the zone center is referred to as the long-wavelength limit. Evaluating the limit

$$
\lim _{k \rightarrow 0} \frac{\partial \omega}{\partial k}=a_{1} \sqrt{\frac{K}{m}}
$$

and noting that both $\omega$ and $k$ equal 0 at the zone center, it is found that

$$
\omega=a \sqrt{\frac{K}{m}} k=c k,
$$

where $c$ is the sound speed in the one-dimensional crystal. In this limit, the wavelength of the elastic waves propagating through the crystal are infinitely long compared to the lattice spacing, and thus, see the crystal as a continuous, rather than discrete medium. 
Keeping this in mind, a common simplification can be made when considering phonon dispersion: the Debye approximation. The Debye approximation was developed under the assumption that a crystalline lattice could be approximated as an elastic continuum. While elastic waves can exist across a range of energies in such a medium, all waves propagate at the same speed. This description exactly mimics the zone center limit described in the previous paragraph, where phonons with wavelengths infinitely long relative to the lattice spacing travel at the sound speed within the crystal. Naturally, then, under the Debye approximation, Eq. 32 holds for phonons of all wavelengths, and hence, all wavevectors. The accuracy of the Debye approximation depends largely on the temperature regime one is working in. In Fig. 3a, both the slopes and the values of the Debye and real dispersion converge at the zone center. As a result, the Debye approximation is most accurate describing phonon transport in the low-temperature limit, where low energy, low frequency phonons dominate (to be discussed in Section 5).

At the zone edge, a second limit can be established and evaluated,

$$
\lim _{k \rightarrow \pi / a} \frac{\partial \omega}{\partial k}=0,
$$

indicating that phonons at the zone edge do not propagate. In this short wavelength limit, the wavelengths of the elastic waves in the crystal are equal to twice the atomic spacing. Here, atoms vibrate entirely out-of-phase with each other, leading to the formation of a standing wave. Advanced texts address the formation of this standing wave further, noting that at $k=\pi / a$, the Bragg reflection condition is satisfied (Srivastava, 1990). Consequently, the coherent scattering and subsequent interference of the incoming wave creates the standing wave condition.

At this point, discussion has been limited to monatomic crystals. However, many materials of technological interest (semiconductors in particular) have polyatomic basis sets. Thus, attention is now given to the diatomic one-dimensional chain illustrated in Fig. $2 b$. Here, $m$ is the mass of the "lighter" atom, and $M$ is the mass of the "heavier" atom, such that $M>m$. Due to the diatomic nature of this system, equation(s) of motion must be formulated for each type of atom in the system,

$$
\begin{aligned}
& m \ddot{u}_{j}=K\left(w_{j}-2 u_{j}+w_{j-1}\right) \\
& m \ddot{w}_{j}=K\left(u_{j+1}-2 w_{j}+u_{j}\right) .
\end{aligned}
$$

Substituting wavelike solutions to these differential equations and isolating $\omega^{2}$ yields

$$
\omega^{2}=K\left(\frac{1}{m}+\frac{1}{M}\right) \pm K\left(\left(\frac{1}{m}+\frac{1}{M}\right)^{2}-\frac{4}{m M} \sin ^{2} k a_{2}\right) .
$$

Perhaps the most unique feature of Eq. 35 is that for each wavevector $k$, two unique values of $\omega$ satisfy the expression. As a result, as the two solutions $\omega_{1}$ and $\omega_{2}$ are plotted against each unique $k$, two distinct phonon branches form: the acoustic branch, and the optical branch.

The distinction between these branches is illustrated in Fig. 3. At the zone center, in the branch of lower energy, atoms $m_{j}$ and $M_{j}$ move in phase with each other, exhibiting the characteristic sound wave behavior discussed above. Thus, this branch is called the acoustic branch. On the other hand, in the branch of higher energy, atoms $m_{j}$ and $M_{j}$ move out of phase with each other. If these atoms had opposite charges on them, as would be the case in an ionic 
crystal, this vibration could be excited by an electric field associated with the infrared edge of visible light spectrum (Srivastava, 1990). As such, this branch is called the optical branch. The phononic band gap between these branches at the zone edge is proportional to the difference in atomic masses (and the effective spring constants). In the unique case where $m=M$, the solution is identical to that of the monatomic chain.

Extending the one-dimensional cases described above to two or three dimensions is conceptually simple, but is often no trivial task. For each atom of the basis set, $n$ equations of motion will be required, where $n$ represents the dimensionality of the system. Generally, solutions for the resulting dispersion diagrams will yield $n$ acoustic branches and $B(n-$ 1) optical branches, where $B$ is the number of atoms comprising the basis. While in the one-dimensional system above we considered only longitudinal modes (compression waves), in three-dimensional systems, two transverse modes will exist as well (shear waves due to atomic displacements in the two directions perpendicular to the direction of wave propagation). Rigorous treatments of such scenarios are presented explicitly in advanced solid-state texts (Srivastava, 1990; Dove, 1993).

\section{Density of states}

A convenient representation of the number of energy states in a solid is through the density of states formulation. The density of states represents the number of states per unit space per unit interval of wavevector or energy. For example, the one-dimensional density of states of electrons represents the number of electron states per unit length per $d k$ or per $d \epsilon$ in the Brillouin zone. Similarly, the three dimensional density of states of phonons represents the number of phonon states per unit volume per $d k$ or per $d \omega$ in the in the Brillouin zone (for phonons $\epsilon=\hbar \omega$ ). The general formulation of the density of states in $n$ dimensions considers the number of states contained in the $n-1$ space of thickness $d k$ per unit space $L^{n}$. Consequently, the density of states has units of states divided by length raised to the $n$ divided by the differential wavevector or energy. For example, the density of states of a three-dimensional solid considers the number of states contained in the volume represented by the two-dimensional surface multiplied by the thickness $d k$ per unit volume $L^{3}$, where $L$ is a length, per $d k$ or $d \epsilon$. In this section, the density of states will be derived for one-, two-, and three-dimensional isotropic solids. The representation of an isotropic solid implies that periodicity arises on a length scale of $k=\pi / a$ and is symmetric about $k=0$, as discussed in the last section. This means, that for the isotropic case considered in this chapter, the total distance from one Brillouin Zone edge to the other is $2 \pi / a$. This general derivation yields a density of states of the $n$-dimensional solid per interval of wavevector given by

$$
D_{n \mathrm{D}}=\frac{(n-1 \text { surface of } n \text {-dimensional space }) d k}{\left(\frac{2 \pi}{a}\right)^{n} L^{n} d k}
$$

or per interval of energy given by

$$
D_{n \mathrm{D}}=\frac{(n-1 \text { surface of } n \text {-dimensional space }) d k}{\left(\frac{2 \pi}{a}\right)^{n} L^{n} d \epsilon},
$$

where $L^{n}$ is the "volume" of unit space $n$. Note that $a^{n}=L^{n}$. In practice, the density of states per interval of energy is more conceptually intuitive and is directly input into expressions for 
the thermal properties, so the starting point for the examples discussed in the remainder of this section will be Eq. 37.

This general density of states formulation can then be recast into energy space via the electron or phonon dispersion relations. This is accomplished by solving the dispersion relation for $k$. For example, the electron dispersion relation, given by Eq. 20, can be rearranged as

$$
k=\sqrt{\frac{2 m \epsilon}{\hbar^{2}}}
$$

and from this

$$
\partial k=\frac{1}{2} \sqrt{\frac{2 m}{\hbar^{2} \epsilon}} \partial \epsilon .
$$

Similarly, assuming the phonon dispersion relation given by Eq. 32 (i.e., the Debye relation) yields

$$
k=\frac{\omega}{v_{g}},
$$

and from this

$$
\partial k=\frac{\partial \omega}{v_{g}} .
$$

Note that recasting Eq. 37 into energy space via a dispersion relation yields the number of states per unit $L^{n}$ per energy interval. In the remainder of this section, the specific derivation of the one-, two- and three-dimensional electron and phonon density of states will be presented. This abstract discussion of the density of states will become much more clear with the specific examples.

\subsection{One-dimensional density of states}

The starting point for the density of states of a one-dimensional system, as generally discussed above, is to consider the number of states in contained in a zero dimensional space multiplied by $d k$ divided by the one-dimensional space of distance $2 \pi / a$. Therefore, the one-dimensional density of states is given by

$$
D_{1 \mathrm{D}}=\frac{d k}{\left(\frac{2 \pi}{a}\right) L d \epsilon} .
$$

From Eq. 39, the one-dimensional electron density of states is given by

$$
D_{\mathrm{e}, 1 \mathrm{D}}=2 \times \frac{a}{2 \pi L d \epsilon} \frac{1}{2} \sqrt{\frac{2 m}{\hbar^{2} \epsilon}} d \epsilon=\frac{1}{2 \pi} \sqrt{\frac{2 m}{\hbar^{2} \epsilon}},
$$

where the subscript e denotes the electron system and the factor of 2 in front of the middle equation arises due to the double degeneracy of the electron states, as discussed in Section 3.1 . From Eq. 41, the one-dimensional phonon density of states is given by

$$
D_{\mathrm{p}, 1 \mathrm{D}}=\frac{a}{2 \pi L \hbar d \omega} \frac{\hbar d \omega}{v_{g}}=\frac{1}{2 \pi v_{g}},
$$

where the subscript $\mathrm{p}$ denotes the phonon system. Since a Debye model is assumed, the phonon group velocity is equal to the speed of sound (i.e., $v_{g}=c$ ), as discussed in Section 3.2. 


\subsection{Two-dimensional density of states}

For the density of states in a two-dimensional (2D) system, the starting point is to consider the number of states along the surface of a circle with radius $k$ multiplied by $d k$ divided by the $2 \mathrm{D}$ space of area $(2 \pi / a)^{2}$. Therefore, the $2 \mathrm{D}$ density of states is given by

$$
D_{2 \mathrm{D}}=\frac{2 \pi k d k}{\left(\frac{2 \pi}{a}\right)^{2} L^{2} d \epsilon} .
$$

From Eq. 38 and 39, the 2D electron density of states is given by

$$
D_{\mathrm{e}, 2 \mathrm{D}}=2 \times \frac{a^{2}}{(2 \pi)^{2} L^{2} d \epsilon} 2 \pi \sqrt{\frac{2 m \epsilon}{\hbar^{2}}} \frac{1}{2} \sqrt{\frac{2 m}{\hbar^{2} \epsilon}} d \epsilon=\frac{1}{\pi} \frac{m}{\hbar^{2}} .
$$

Note that the 2D density of states for electrons is independent of energy. From Eq. 40 and 41, the $2 \mathrm{D}$ phonon density of states is given by

$$
D_{\mathrm{p}, 2 \mathrm{D}}=2 \times \frac{a^{2}}{(2 \pi)^{2} L^{2} \hbar d \omega} 2 \pi \frac{\omega}{v_{g}} \frac{\hbar d \omega}{v_{g}}=\frac{\omega}{\pi v_{g}^{2}} .
$$

where the factor of 2 in front of the middle equation arises due to the second dimension, which introduces a transverse polarization in addition to the longitudinal polarization, as discussed in Section 3.2. In the discussions in this chapter, equal phonon velocities and frequencies (i.e., dispersions) are assumed for each phonon polarization.

\subsection{Three-dimensional density of states}

The density of states in three-dimensions (3D) will be extensively used in the remainder of this chapter to discuss nanoscale thermal processes. Following the previous discussions in this section, the 3D density of states is formulated by considering the the number of states contained on the surface of a sphere in $k$-space multiplied by the thickness of the sphere $d k$ divided by the 3D space of volume $(2 \pi / a)^{3}$. Therefore, the 3D density of states is given by

$$
D_{3 \mathrm{D}}=\frac{4 \pi k^{2} d k}{\left(\frac{2 \pi}{a}\right)^{3} L^{3} d \epsilon} .
$$

From Eq. 38 and 39, the 3D electron density of states is given by

$$
D_{\mathrm{e}, 3 \mathrm{D}}=2 \times \frac{a^{3}}{(2 \pi)^{3} L^{3} d \epsilon} 4 \pi \frac{2 m \epsilon}{\hbar^{2}} \frac{1}{2} \sqrt{\frac{2 m}{\hbar^{2} \epsilon}} d \epsilon=\frac{1}{2 \pi^{2}}\left(\frac{2 m}{\hbar^{2}}\right)^{\frac{3}{2}} \epsilon^{\frac{1}{2}} .
$$

From Eq. 40 and 41, the 3D phonon density of states is given by

$$
D_{\mathrm{p}, 3 \mathrm{D}}=3 \times \frac{a^{3}}{(2 \pi)^{3} L^{3} \hbar d \omega} 4 \pi \frac{\omega^{2}}{v_{g}^{2}} \frac{\hbar d \omega}{v_{g}}=\frac{3 \omega^{2}}{2 \pi^{2} v_{g}^{3}},
$$

where the factor of 3 in front of the middle equation arises due to the three dimensions, which introduces two additional transverse polarizations along with the longitudinal polarization, as discussed in Section 3.2. 


\section{Statistical mechanics}

The principles of quantum mechanics discussed in the previous two sections give the allowable energy states of electrons and phonons. However, this development did not discuss the way in which these thermal energy carriers can occupy the quantum states. The bridge connecting the allowable and occupied quantum states to the collective behavior of the energy carriers in a nanosystem is provided by statistical mechanics. Through statistical mechanics, temperature enters into the picture and physical properties such as internal energy and heat capacity are defined.

It turns out that the thermal energy carriers in nature divide into two classes, fermions and bosons, which differ in the way they can occupy their respective density of states. Electrons are fermions that follow a rule that only one particle can occupy a fully described quantum state (where there are two quantum states with different spins per energy, as discussed in Section 3.1). This rule was first recognized by Pauli and is called the Pauli exclusion principle. In a system with many states and many fermion particles to fill these states, particles first fill the lowest energy states, increasing in energy until all particles are placed. As previously discussed in Section 3.1, the highest filled energy is called the Fermi energy, $\epsilon_{\mathrm{F}}$. Phonons are bosons and are not governed by the Pauli exclusion principle. Any number of phonons can fall into exactly the same quantum state.

When a nanophysical system is in equilibrium with a thermal environment at temperature $T$, then average occupation expectation values for the quantum states are found to exist. In the case of electrons (fermions), the occupation function is the Fermi-Dirac distribution function, given by

$$
f_{\mathrm{FD}}=\frac{1}{\exp \left[\frac{\epsilon-\epsilon_{\mathrm{F}}}{k_{\mathrm{B}} T}\right]+1},
$$

where $k_{\mathrm{B}}$ is Boltzmann's constant (Boltzmann's constant is $k_{\mathrm{B}}=1.3807 \times 10^{-23} \mathrm{JK}^{-1}$ ). For phonons (bosons), the corresponding occupation function is the Bose-Einstein distribution function, given by

$$
f_{\mathrm{BE}}=\frac{1}{\exp \left[\frac{\hbar \omega}{k_{\mathrm{B}} T}\right]-1} .
$$

Figure $4 \mathrm{a}$ and $\mathrm{b}$ show plots of Eqs. 51 as a function of electron energy and 52 as a function phonon frequency, respectively, for three different temperatures, $T=10,500$, and $1000 \mathrm{~K}$.

Given the distribution of carriers, the number of electrons/phonons in a bulk solid at a given temperature is defined as

$$
n_{\mathrm{e} / \mathrm{p}}=\int_{\epsilon} D_{\mathrm{e} / \mathrm{p}} f_{\mathrm{FD} / \mathrm{BE}} d \epsilon,
$$

where the dimensionality of the system is driven by the dimensionality of the density of states of the electrons or phonons derived in Section 4 . The total number of electrons and phonons is mathematically expressed by Eq. 53. The total number of electrons in a bulk solid is constant as the Fermi-Dirac distribution only varies between zero and one, as seen in Fig. 4a; this is also conceptually a consequence of the Pauli exclusion principle previously mentioned. Although the distribution of electron energies change, the number density stays the same. The phonon number density, however, which has no restriction on number of phonons per quantum states, continues to increase with increasing temperature. Note that at low temperatures, the majority of the phonons exist at low frequencies (low energy/long wavelengths). These phonons correspond to phonons near the center of the Brillouin zone $(k=0)$. As temperature 

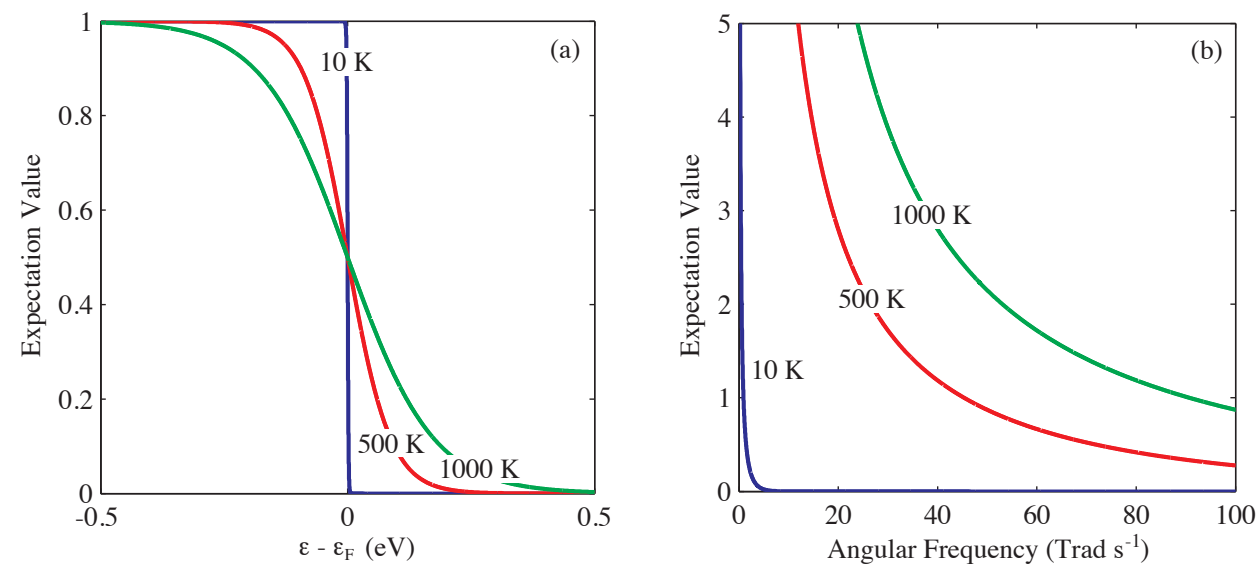

Fig. 4. (a) Fermi-Dirac and (b) Bose-Einstein expectation values calculated from Eqs. 51 and 52 , respectively, for three different temperatures, $T=10,500$, and $1000 \mathrm{~K}$. Note that the expectations values of the Fermi-Dirac distribution function vary from zero to unity, and therefore represent the probability of an electron being at a certain energy state.

is increased, the proportion of higher frequency (higher energy/shorter wavelength) phonons that exist increases; these phonons correspond to phonons that are closer to the Brillouin zone edge $(k=\pi / a)$. With the number of electrons/phonons defined in Eq. 53 and following the discussion in Section 2, the internal energy of the electron/phonon system is defined as

$$
U_{\mathrm{e} / \mathrm{p}}=\int_{\epsilon} \epsilon D_{\mathrm{e} / \mathrm{p}} f_{\mathrm{FD} / \mathrm{BE}} d \epsilon .
$$

Now that the internal energies of the electrons and phonons are defined in terms of the properties of the individual energy carriers, their correspond heat capacities are given by the temperature derivative of the internal energies, as discussed in Section 2; that is,

$$
C=\frac{\partial U}{\partial T}
$$

The heat capacities of electrons and phonons for one-, two-, and three-dimensional solids will be studied in the remainder of this section.

\subsection{Electron heat capacity}

Since the zero temperature state of a free electron gas does not correspond to a zero internal energy system (i.e., $U(T=0) \neq 0)$ ), care must be taken when defining the integration limits in the calculation of the heat capacity. To begin, the internal energy of the $T=0$ state of a free electron gas is given by

$$
U_{\mathrm{e}}(T=0)=\int_{0}^{\epsilon_{\mathrm{F}}} \epsilon D_{\mathrm{e}} f_{\mathrm{FD}} d \epsilon .
$$


As temperature increases, the electrons redistribute themselves to higher energy levels and the internal energy is calculated by considering electrons over all energy states, given by

$$
U_{\mathrm{e}}(T=0)=\int_{0}^{\infty} \epsilon D_{\mathrm{e}} f_{\mathrm{FD}} d \epsilon
$$

Therefore, the change in internal energy of the electron system given some arbitrary $\delta T$ is determined by subtracting Eq. 56 from 57, yielding

$$
\delta U_{\mathrm{e}}=\int_{0}^{\infty}\left(\epsilon-\epsilon_{\mathrm{F}}\right) D_{\mathrm{e}}\left(\delta f_{\mathrm{FD}}\right) d \epsilon .
$$

Following Eq. 55, the electronic heat capacity is given by

$$
C_{\mathrm{e}}=\int_{0}^{\infty}\left(\epsilon-\epsilon_{\mathrm{F}}\right) D_{\mathrm{e}} \frac{\partial f_{\mathrm{FD}}}{\partial T} d \epsilon
$$

At this point, the various electronic density of states defined in Section 4 will be inserted into Eq. 59 to study the effects of dimensionality on electronic thermal storage. For convenience, the electronic heat capacity discussion will be limited to metals since electrons are the dominant thermal carriers in metals and convenient simplifications in the heat capacity derivations can be made to elucidate the interesting thermophysics. Mainly, at low-to-moderate temperatures, the density of states in metals can be considered constant and evaluated at the Fermi energy. This simplifying assumption means that the density of states can be taken out of the integral in Eq. 59. Therefore, Eq. 59 can be rewritten as

$$
C_{\mathrm{e}}=D_{\mathrm{e}}\left(\epsilon_{\mathrm{F}}\right) \int_{0}^{\infty}\left(\epsilon-\epsilon_{\mathrm{F}}\right) \frac{\partial f_{\mathrm{FD}}}{\partial T} d \epsilon=D_{\mathrm{e}}\left(\epsilon_{\mathrm{F}}\right) \int_{0}^{\infty} \frac{\left(\epsilon-\epsilon_{\mathrm{F}}\right)^{2}}{k_{\mathrm{B}} T^{2}} \frac{\exp \left(\frac{\epsilon-\epsilon_{\mathrm{F}}}{k_{\mathrm{B}} T}\right)}{\left(\exp \left(\frac{\epsilon-\epsilon_{\mathrm{F}}}{k_{\mathrm{B}} T}\right)+1\right)^{2}} d \epsilon .
$$

Making the substitution of $x \equiv\left(\epsilon-\epsilon_{\mathrm{F}}\right) /\left(k_{\mathrm{B}} T\right)$, Eq. 60 can be re-expressed as

$$
C_{\mathrm{e}}=D_{\mathrm{e}}\left(\epsilon=\epsilon_{\mathrm{F}}\right) k_{\mathrm{B}} T^{2} \int_{-\frac{\epsilon_{\mathrm{F}}}{k_{\mathrm{B}} T}}^{\infty} x^{2} \frac{\exp (x)}{(\exp (x)+1)^{2}} d x .
$$

To simplify this integral, consider the lower bound of $-\epsilon_{\mathrm{F}} /\left(k_{\mathrm{B}} T\right)$. At low to moderate temperatures, the magnitude of this quantity is very large, meaning that this lower bound extends to very large negative numbers. Therefore, the lower bound of Eq. 61 can be approximated as negative infinity, so that Eq. 61 can be recast as

$$
C_{\mathrm{e}}=D_{\mathrm{e}}\left(\epsilon=\epsilon_{\mathrm{F}}\right) k_{\mathrm{B}} T^{2} \int_{-\infty}^{\infty} x^{2} \frac{\exp (x)}{(\exp (x)+1)^{2}} d x
$$

This integral can now be solved exactly. By recognizing that

$$
\int_{-\infty}^{\infty} x^{2} \frac{\exp (x)}{(\exp (x)+1)^{2}} d x=\frac{\pi^{2}}{3}
$$


the electronic heat capacity is given by

$$
C_{\mathrm{e}}=\frac{\pi^{2}}{3} k_{\mathrm{B}}^{2} T D_{\mathrm{e}}\left(\epsilon=\epsilon_{\mathrm{F}}\right) .
$$

Now to study the electronic heat capacity of electronic systems with different dimensionalities, the various electronic densities of states derived in Section 4 just need be inserted into Eq. 64.

Consider the 3D electron density of states given by Eq. 49. Plugging this into Eq. 64 yields

$$
C_{\mathrm{e}, 3 \mathrm{D}}=\frac{\pi^{2}}{3} k_{\mathrm{B}}^{2} T \frac{1}{2 \pi^{2}}\left(\frac{2 m}{\hbar^{2}}\right)^{\frac{3}{2}} \epsilon_{\mathrm{F}}^{\frac{1}{2}}=\frac{k_{\mathrm{B}}^{2} T}{6}\left(\frac{2 m}{\hbar^{2}}\right)^{\frac{3}{2}} \epsilon_{\mathrm{F}}^{\frac{1}{2}} .
$$

To simplify this expression further, consider Eq. 53 for a 3D system of electrons. Since, as previously mentioned, Eq. 53 is constant for electrons, this expression can be evaluated exactly at $T=0$ to give analytical expression for the electron number density. At zero temperature, Eq. 53 for electrons becomes

$$
n_{\mathrm{e}, 3 \mathrm{D}}=\int_{0}^{\epsilon_{\mathrm{F}}} D_{\mathrm{e}, 3 \mathrm{D}} f_{\mathrm{FD}}(T=0) d \epsilon=\int_{0}^{\epsilon_{\mathrm{F}}} D_{\mathrm{e}, 3 \mathrm{D}} d \epsilon=\frac{1}{3 \pi^{2}}\left(\frac{2 m}{\hbar^{2}}\right)^{\frac{3}{2}} \epsilon_{\mathrm{F}}^{\frac{3}{2}} .
$$

and from this, it is apparent that for free electrons in a 3D metallic system

$$
\left(\frac{2 m}{\hbar^{2}}\right)^{\frac{3}{2}}=\frac{3 \pi^{2} n_{\mathrm{e}, 3 \mathrm{D}}}{\epsilon_{\mathrm{F}}^{\frac{3}{2}}}
$$

Inserting Eq. 67 in 65 yields

$$
C_{\mathrm{e}, 3 \mathrm{D}}=\frac{\pi^{2} k_{\mathrm{B}}^{2} n_{\mathrm{e}, 3 \mathrm{D}}}{2 \epsilon_{\mathrm{F}}} T,
$$

showing that for a 3D system of free electrons, the heat capacity is directly related to the temperature, where the proportionality constant is related to material properties. The electronic heat capacity of Au is plotted in Fig 5.

To examine the electronic heat capacity of a $2 \mathrm{D}$ electronic system, consider the $2 \mathrm{D}$ electron density of states given by Eq. 46. Substituting this 2D density of states into Eq. 64 yields

$$
C_{\mathrm{e}, 2 \mathrm{D}}=\frac{\pi^{2}}{3} k_{\mathrm{B}}^{2} T \frac{1}{\pi} \frac{m}{\hbar^{2}}=\frac{\pi k_{\mathrm{B}}^{2} T}{3} \frac{m}{\hbar^{2}} .
$$

Following the development for the 3D heat capacity, Eq. 53 for a $2 \mathrm{D}$ system of electrons is given by

$$
n_{\mathrm{e}, 2 \mathrm{D}}=\int_{0}^{\epsilon_{\mathrm{F}}} D_{\mathrm{e}, 2 \mathrm{D}} f_{\mathrm{FD}}(T=0) d \epsilon=\int_{0}^{\epsilon_{\mathrm{F}}} D_{\mathrm{e}, 2 \mathrm{D}} d \epsilon=\frac{1}{\pi} \frac{m}{\hbar^{2}} \epsilon_{\mathrm{F}} .
$$

From this, it is apparent that for free electrons in a 2D metallic system

$$
\frac{m}{\hbar^{2}}=\frac{\pi n_{\mathrm{e}, 2 \mathrm{D}}}{\epsilon_{\mathrm{F}}} .
$$


Inserting Eq. 71 in 69 yields

$$
C_{\mathrm{e}, 2 \mathrm{D}}=\frac{\pi^{2} k_{\mathrm{B}}^{2} n_{\mathrm{e}, 2 \mathrm{D}}}{3 \epsilon_{\mathrm{F}}} T,
$$

which has a similar dependence on temperature and material properties as the electronic heat capacity in 3D.

Finally, for a one-dimensional electronic system, consider the one-dimensional density of states given by Eq. 43. Plugging this into Eq. 64 yields

$$
C_{\mathrm{e}, 1 \mathrm{D}}=\frac{\pi^{2}}{3} k_{\mathrm{B}}^{2} T \frac{1}{2 \pi} \sqrt{\frac{2 m}{\hbar^{2} \epsilon_{\mathrm{F}}}}=\frac{\pi}{6} k_{\mathrm{B}}^{2} T \sqrt{\frac{2 m}{\hbar^{2} \epsilon_{\mathrm{F}}}} .
$$

The number density of a one-dimensional system of electrons is given by

$$
n_{\mathrm{e}, 1 \mathrm{D}}=\int_{0}^{\epsilon_{\mathrm{F}}} D_{\mathrm{e}, 1 \mathrm{D}} f_{\mathrm{FD}}(T=0) d \epsilon=\int_{0}^{\epsilon_{\mathrm{F}}} D_{\mathrm{e}, 1 \mathrm{D}} d \epsilon=\frac{1}{2 \pi} \sqrt{\frac{2 m \epsilon_{\mathrm{F}}}{\hbar^{2}}} .
$$

From this

$$
\sqrt{\frac{2 m}{\hbar^{2}}}=\frac{2 \pi n_{\mathrm{e}, 1 \mathrm{D}}}{\sqrt{\epsilon_{\mathrm{F}}}}
$$

which yields

$$
C_{\mathrm{e}, 1 \mathrm{D}}=\frac{\pi^{2} k_{\mathrm{B}}^{2} n_{\mathrm{e}, 1 \mathrm{D}}}{3 \epsilon_{\mathrm{F}}} T,
$$

which is also directly proportional to temperature. As apparent from the derivations of the electronic heat capacities in different dimensionalities of electron systems, the electronic heat capacity is always directly related to the temperature, regardless of the electron system dimension.

\subsection{Phonon heat capacity}

Unlike electrons (fermions), the zero temperature state of phonons (bosons) does not correspond to a zero internal energy state (i.e., $U(T=0) \neq 0$ ) since at $T=0$, the lattice is not vibrating so phonons do not exist. Therefore, the change in internal energy of the phonon system given some arbitrary $\delta \mathrm{T}$ is determined by evaluating

$$
\delta U_{\mathrm{p}}=\int_{0}^{\omega_{\max }} \hbar \omega D_{\mathrm{p}}\left(\delta f_{\mathrm{BE}}\right) d \omega
$$

Following Eq. 55, the phonon heat capacity is given by

$$
C_{\mathrm{p}}=\int_{0}^{\omega_{\max }} \hbar \omega D_{\mathrm{p}} \frac{\partial f_{\mathrm{BE}}}{\partial T} d \omega=\int_{0}^{\omega_{\max }} \frac{\hbar^{2} \omega^{2}}{k_{\mathrm{B}} T^{2}} D_{\mathrm{p}} \frac{\exp \left[\frac{\hbar \omega}{k_{\mathrm{B}} T}\right]}{\left(\exp \left[\frac{\hbar \omega}{k_{\mathrm{B}} T}\right]-1\right)^{2}} d \omega .
$$

Since the Debye assumption is employed for the phonon dispersion in these examples, the maximum phonon frequency is defined as $\omega_{\max }=v_{g} \pi / a_{1}$. 
The 3D phonon heat capacity is derived by plugging in the expression for the $3 \mathrm{D}$ phonon density of states (Eq. 50) in Eq. 78 which gives

$$
C_{\mathrm{p}, 3 \mathrm{D}}=\int_{0}^{\omega_{\max }} \frac{\hbar^{2} \omega^{2}}{k_{\mathrm{B}} T^{2}} \frac{3 \omega^{2}}{2 \pi^{2} v_{g}^{3}} \frac{\exp \left[\frac{\hbar \omega}{k_{\mathrm{B}} T}\right]}{\left(\exp \left[\frac{\hbar \omega}{k_{\mathrm{B}} T}\right]-1\right)^{2}} d \omega=\int_{0}^{\omega_{\max }} \frac{3 \hbar^{2} \omega^{4}}{2 \pi^{2} v_{g}^{3} k_{\mathrm{B}} T^{2}} \frac{\exp \left[\frac{\hbar \omega}{k_{\mathrm{B}} T}\right]}{\left(\exp \left[\frac{\hbar \omega}{k_{\mathrm{B}} T}\right]-1\right)^{2}} d \omega .
$$

The 3D phonon heat capacity of Au is plotted in Fig. 5 along with the electronic heat capacity. Note that the phonon system heat capacity approaches a constant values at high temperatures. This limit of constant phonon heat capacity is called the Dulong and Petit limit. The onset of this Dulong and Petit limit (i.e., the onset of the constant phonon heat capacity) occurs around a material property called the Debye temperature. The Debye temperature is approximately the equivalent temperature in which all phonon modes in a solid are excited; this Debye temperature concept will be quantified in more detail below. Also, note that at very low temperatures $(T \approx 1 \mathrm{~K})$, the electron system heat capacity is larger than that of the phonon system. However, for the majority of the temperature range in which Au is solid (the melting temperature of gold is about 1,300 K), the phonon heat capacity is several orders of magnitude larger than that of the electrons. Note also the low temperature trend of the phonon heat capacity is different than the liner trend in temperature exhibited by the electron system. For the remainder of this section, the low temperature trends in the phonon heat capacity, and the effect of dimensionality on this trend, will be explored.

To examine the low temperature trends in phonon heat capacity, it is convenient to make the variable substitution $x \equiv \hbar \omega /\left(k_{\mathrm{B}} T\right)$. With this, the 3D phonon heat capacity becomes

$$
C_{\mathrm{p}, 3 \mathrm{D}}=\frac{3 k_{\mathrm{B}}^{4}}{2 \pi^{2} v_{g}^{3} \hbar^{3}} T^{3} \int_{0}^{x_{\max } \equiv \theta_{\mathrm{D}} / T} x^{4} \frac{\exp [x]}{(\exp [x]-1)^{2}} d x
$$

where the upper limit is redefined as the Debye temperature, $\theta_{\mathrm{D}}$, divided by the temperature. Note that $\theta_{\mathrm{D}}=\hbar \omega_{\max } / k_{\mathrm{B}}$, which is, as previously conceptually discussed, directly related to the maximum phonon frequency in a solid. In this low temperature limit, $T \ll \theta_{\mathrm{D}}$ and $x_{\max } \longrightarrow \infty$, so that the integral in Eq. 80 can be evaluated exactly. Recognizing that

$$
\int_{0}^{\infty} x^{4} \frac{\exp [x]}{(\exp [x]-1)^{2}} d x=\frac{4 \pi^{4}}{15}
$$

the low temperature heat capacity in a $3 \mathrm{D}$ phonon system becomes

$$
C_{\mathrm{p}, 3 \mathrm{D}}=\frac{2 \pi^{2} k_{\mathrm{B}}^{4}}{5 v_{g}^{3} \hbar^{3}} T^{3}
$$

showing that for a 3D system of phonons, the heat capacity is directly related to the cube of the temperature at low temperatures, where the proportionality constant is related to material properties.

Following a similar derivation for a 2D phonon system, plugging Eq. 47 in Eq. 78 gives

$$
C_{\mathrm{p}, 2 \mathrm{D}}=\int_{0}^{\omega_{\max }} \frac{\hbar^{2} \omega^{2}}{k_{\mathrm{B}} T^{2}} \frac{\omega}{\pi v_{g}^{2}} \frac{\exp \left[\frac{\hbar \omega}{k_{\mathrm{B}} T}\right]}{\left(\exp \left[\frac{\hbar \omega}{k_{\mathrm{B}} T}\right]-1\right)^{2}} d \omega=\int_{0}^{\omega_{\max }} \frac{\hbar^{2} \omega^{3}}{\pi v_{g}^{2} k_{\mathrm{B}} T^{2}} \frac{\exp \left[\frac{\hbar \omega}{k_{\mathrm{B}} T}\right]}{\left(\exp \left[\frac{\hbar \omega}{k_{\mathrm{B}} T}\right]-1\right)^{2}} d \omega .
$$




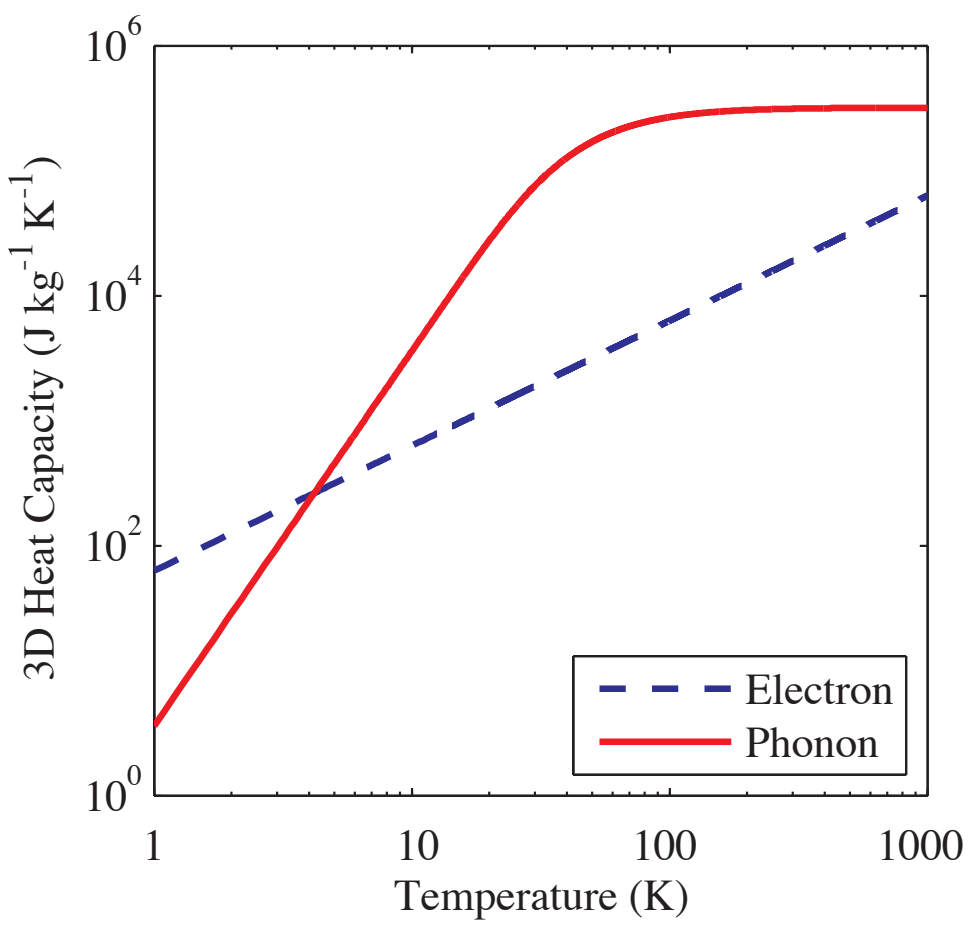

Fig. 5. 3D electron and phonon heat capacities of Au calculated from Eq. 68 and 80, respectively. For these calculations, the Au material parameters are assumed as $n_{\mathrm{e}, 3 \mathrm{D}}=5.9 \times 10^{28} \mathrm{~m}^{-3}, \epsilon_{\mathrm{F}}=5.5 \mathrm{eV}=8.811 \times 10^{-19} \mathrm{~J}$, and $v_{\mathrm{g}}=3,240 \mathrm{~m} \mathrm{~s}^{-1}$.

Making the above mentioned $x$-substitution yields

$$
C_{\mathrm{p}, 2 \mathrm{D}}=\frac{k_{\mathrm{B}}^{3}}{\operatorname{piv}_{g}^{2} \hbar^{2}} T^{2} \int_{0}^{\theta_{\mathrm{D}} / T} x^{3} \frac{\exp [x]}{(\exp [x]-1)^{2}} d x,
$$

As with the 3D case, at low temperatures, the integration can be extended to infinity. Recognizing that

$$
\int_{0}^{\infty} x^{3} \frac{\exp [x]}{(\exp [x]-1)^{2}} d x=6 \zeta[3],
$$

where $\zeta[3]$ is the Zeta function evaluated at 3, the low temperature heat capacity in a $2 \mathrm{D}$ phonon system becomes

$$
C_{\mathrm{p}, 2 \mathrm{D}}=\frac{6 \zeta[3] k_{\mathrm{B}}^{3}}{\pi v_{g}^{2} \hbar^{2}} T^{2},
$$

showing that for a 2D system of phonons, the heat capacity is directly related to the square of the temperature at low temperatures, where the proportionality constant is related to material properties. 
Following a above derivations, the heat capacity of a one-dimensional phonon system is derived by plugging Eq. 44 in Eq. 78 which gives

$$
C_{\mathrm{p}, 1 \mathrm{D}}=\int_{0}^{\omega_{\max }} \frac{\hbar^{2} \omega^{2}}{k_{\mathrm{B}} T^{2}} \frac{1}{2 \pi v_{g}} \frac{\exp \left[\frac{\hbar \omega}{k_{\mathrm{B}} T}\right]}{\left(\exp \left[\frac{\hbar \omega}{k_{\mathrm{B}} T}\right]-1\right)^{2}} d \omega=\int_{0}^{\omega_{\max }} \frac{\hbar^{2} \omega^{2}}{2 \pi v_{g} k_{\mathrm{B}} T^{2}} \frac{\exp \left[\frac{\hbar \omega}{k_{\mathrm{B}} T}\right]}{\left(\exp \left[\frac{\hbar \omega}{k_{\mathrm{B}} T}\right]-1\right)^{2}} d \omega .
$$

Making the above mentioned $x$-substitution yields

$$
C_{\mathrm{p}, 1 \mathrm{D}}=\frac{k_{\mathrm{B}}^{2}}{2 \pi v_{g} \hbar} T \int_{0}^{\theta_{\mathrm{D}} / T} x^{2} \frac{\exp [x]}{(\exp [x]-1)^{2}} d x
$$

As with the previous cases, at low temperatures, the integration can be extended to infinity. Recognizing that

$$
\int_{0}^{\infty} x^{2} \frac{\exp [x]}{(\exp [x]-1)^{2}} d x=\frac{\pi^{2}}{3}
$$

the low temperature heat capacity in a one-dimensional phonon system becomes

$$
C_{\mathrm{p}, 1 \mathrm{D}}=\frac{\pi k_{\mathrm{B}}^{2}}{6 v_{g} \hbar} T
$$

showing that for a one-dimensional system of phonons, the heat capacity is directly and linearly related to the the temperature at low temperatures, where the proportionality constant is related to material properties. Note that, unlike the electron systems which in which the temperature trend in heat capacity does not change with dimensionality, an $n$-dimensional phonon system has a temperature dependency of $T^{n}$.

\section{Thermal conductivity}

In the preceding sections, the quantum energy states of electrons and phonons were derived, and from this, expressions for heat capacities of these thermal energy carriers were presented. With this, given a particle velocity and scattering time, the thermal conductivity can be calculated via Eq. 6. In this final section, the thermal conductivity of electrons and phonons will be calculated from the quantum derivations of heat capacity. The discussion will be limited to systems in which a 3D density of states can still be assumed and the electrons and phonons are treated as particles experiencing scattering events, as in the Kinetic Theory of Gases discussion in Section 2 This approximation of particle transport typical holds true until characteristic dimensions of nanosystems get below about $10 \mathrm{~nm}$ at elevated temperatures $(T>50 K)$. Taking the particle approach, and referring to Eq. 6, the thermal conductivity is given by

$$
\kappa_{\mathrm{e} / \mathrm{p}}=\frac{1}{3} C_{\mathrm{e} / \mathrm{p}, 3 \mathrm{D}} v_{\mathrm{e} / \mathrm{p}}^{2} \tau_{\mathrm{e} / \mathrm{p}}=\int_{\epsilon} \epsilon D_{\mathrm{e} / \mathrm{p}} \frac{\partial f_{\mathrm{FD} / \mathrm{BE}}}{\partial T} v_{\mathrm{e} / \mathrm{p}}^{2} \tau_{\mathrm{e} / \mathrm{p}} d \epsilon
$$

As previously discussed, electrons are the dominant thermal carrier in metals where phonons are the dominant thermal carrier in semiconductors; therefore, the derivation of electron thermal conductivity will focus on gold for example calculations and the phonon thermal conductivity calculations will focus on silicon. 
The final two quantities needed to determine the thermal conductivity of electrons and phonons are their respective scattering times and velocities. In our particle treatment, the electrons and phonons can scatter via several different mechanisms, which will be discussed in more detail later in this section. However, the total scattering rate used in Eq. 91 is related to the individual scattering processes of each thermal carrier via Matthiessen's Rule, given by (Kittel, 2005)

$$
\frac{1}{\tau}=\sum_{m} \frac{1}{\tau_{m}}
$$

where $m$ is an index representing a specific scattering process of an electron or a phonon. As for the velocities of the carriers, the phonon velocity was previous defined in Section 3.2, specifically Eq. 30. Typical phonon group velocities are on the order of $v_{g}=10^{3}-10^{4} \mathrm{~m} \mathrm{~s}^{-1}$. The electron velocities can be calculated from the Fermi energy. As the electronic thermal conductivity is related to the temperature derivative of the Fermi-Dirac distribution, only electrons around the Fermi energy will participate in transport. Approximating all the electrons participating in transport to have energies of about the Fermi energy, the velocity of the electrons at the Fermi energy, the Fermi velocity, can be calculated from the common expression for kinetic energy of a particle so that the electron Fermi velocity is given by

$$
v_{\mathrm{F}}=\sqrt{\frac{2 \epsilon_{\mathrm{F}}}{m}} .
$$

Typical Fermi velocities in metals are on the order of $10^{6} \mathrm{~m} \mathrm{~s}^{-1}$.

\subsection{Electron thermal conductivity}

To calculate the thermal conductivity of the electron system via Eq. 91, the final piece of information that must be known is the electron scattering time. At moderate temperatures, electrons can lose energy by scattering with other electrons and with the phonons. In metals, the electron-electron and electron-phonon scattering processes take the form $\tau_{\mathrm{ee}}=\left(A_{\mathrm{ee}} T^{2}\right)^{-1}$ and $\tau_{\mathrm{ep}}=\left(B_{\mathrm{ep}} T\right)^{-1}$, respectively, where $A$ and $B$ are material dependent constants related to the electrical resistivity (Kittel, 2005). From Eq. 94, the total scattering time at moderate temperatures in metals is given by

$$
\frac{1}{\tau}=\left(\frac{1}{\tau_{\mathrm{ee}}}+\frac{1}{\tau_{\mathrm{ep}}}\right)=A_{\mathrm{ee}} T^{2}+B_{\mathrm{ep}} T .
$$

From this, the electron thermal conductivity is given by

$$
\kappa_{\mathrm{e}}=\frac{v_{\mathrm{F}}^{2}}{A_{\mathrm{ee}} T^{2}+B_{\mathrm{ep}} T} \int_{-\infty}^{\infty}\left(\epsilon-\epsilon_{\mathrm{F}}\right) D_{\mathrm{e}, 3 \mathrm{D}}\left(\epsilon_{\mathrm{F}}\right) \frac{\partial f_{\mathrm{FD}}}{\partial T} d \epsilon=\frac{\pi^{2} k_{\mathrm{B}}^{2} n_{\mathrm{e}, 3 \mathrm{D}} v_{\mathrm{F}}^{2}}{2 \epsilon_{\mathrm{F}}\left(A_{\mathrm{ee}} T+B_{\mathrm{ep}}\right)},
$$

where the simplification on the right hand side comes from the development in Section 5.1. The electron thermal conductivity of $\mathrm{Au}$ as a function of temperature predicted via Eq. 95 is shown in Fig. 6a along with the data from Fig. 1. Since the forms of the scattering times in metals discussed above are only valid for temperatures around and above the Debye temperature, the thermal conductivity is shown in the range from $100-1000 \mathrm{~K}$. Below this range, additional electron and phonon iterations affect the conductivity that are beyond the scope of this chapter. The scattering constants, $A_{\text {ee }}$ and $B_{\text {ep }}$ are used to fit the model in Eq. 95 
to the data, and the resulting constants, listed in the figure caption, are in excellent agreement with previously published values (Ivanov \& Zhigilei, 2003). In addition, the temperature trends agree remarkably well even with the simplified assumptions involved in the derivation of Eq. 95, showing the power of modeling electron thermal transport from a fundamental particle level.

With this approach, the effects of nanostructuring on thermal conductivity can now be calculated. When the sizes of a nanomaterial are on the same order as the mean free path of the thermal carriers, in the case of metals, the electrons, an additional scattering mechanism arises due to electron boundary scattering. This boundary scattering time is related to the length of the limiting dimension, $d$, in the nanosystem through $\tau_{\mathrm{eb}}=d / v_{\mathrm{F}}$. Using this with Matthiessen's Rule (Eq. 94), the thermal conductivity of a metallic nanosystem can be calculated by (Hopkins et al., 2008)

$$
\kappa_{\mathrm{e}}=\frac{\pi^{2} k_{\mathrm{B}}^{2} n_{\mathrm{e}, 3 \mathrm{D}} v_{\mathrm{F}}^{2} T}{2 \epsilon_{\mathrm{F}}\left(A_{\mathrm{ee}} T^{2}+B_{\mathrm{ep}} T+\frac{v_{\mathrm{F}}}{d}\right)} .
$$

Note that when $d$ is very large, Eq. 96 reduces to Eq. 95. Fig. 6a shows the predicted thermal conductivity as a function of temperature for Au nanosystems with limiting $d$ indicated in the figure. Due to electron-boundary scattering, the thermal conductivity of metallic nanosystems can be greatly reduced by nanostructuring.

\subsection{Phonon thermal conductivity}

As with the electron thermal conductivity, to calculate the thermal conductivity of the phonon system via Eq. 91, the phonon scattering times must be known. The major phonon scattering processes, valid at all temperatures, are phonon-phonon scattering, phonon-impurity scattering, and phonon-boundary scattering. Note that phonon boundary scattering exists even in bulk samples since phonons exist as a spectrum of wavelengths, some of which can be larger than bulk samples. These processes take the form of $\tau_{\mathrm{pp}}=$ $\left(A T \omega^{2} \exp [-B / T]\right)^{-1}$ for phonon-phonon scattering, $\tau_{\mathrm{pi}}=\left(C \omega^{4}\right)^{-1}$ for phonon-impurity scattering, and $\tau_{\mathrm{pb}}=\left(v_{g} / d\right)^{-1}$ for phonon-boundary scattering. Note that this boundary scattering term represents the bulk boundaries. From this, the total scattering time for phonons is given by

$$
\frac{1}{\tau}=\left(\frac{1}{\tau_{\mathrm{pp}}}+\frac{1}{\tau_{\mathrm{pi}}}+\frac{1}{\tau_{\mathrm{pb}}}\right)=A T \omega^{2} \exp \left[-\frac{B}{T}\right]+C \omega^{4}+\frac{v_{g}}{d} .
$$

and the phonon thermal conductivity can be calculated via

$$
\begin{gathered}
\kappa_{\mathrm{e}}=\int_{0}^{\omega_{\max }} \hbar \omega D_{\mathrm{p}, 3 \mathrm{D}} \frac{\partial f_{\mathrm{BE}}}{\partial T} v_{g}^{2}\left(A T \omega^{2} \exp \left[-\frac{B}{T}\right]+C \omega^{4}+\frac{v_{g}}{d}\right)^{-1} d \omega \\
=\int_{0}^{\omega_{\max }} \frac{3 \hbar^{2} \omega^{4}}{2 \pi^{2} v_{g} k_{\mathrm{B}} T^{2}} \frac{\exp \left[\frac{\hbar \omega}{k_{\mathrm{B}} T}\right]}{\left(\exp \left[\frac{\hbar \omega}{k_{\mathrm{B}} T}\right]-1\right)^{2}}\left(A T \omega^{2} \exp \left[-\frac{B}{T}\right]+C \omega^{4}+\frac{v_{g}}{d}\right)^{-1} d \omega .
\end{gathered}
$$

where the simplification on the right hand side comes from the development in Section 5.2. The phonon thermal conductivity of $\mathrm{Si}$ as a function of temperature predicted via Eq. 98 is shown in Fig. $6 \mathrm{~b}$ along with the data from Fig. 1 . The scattering time coefficients $A$ and 

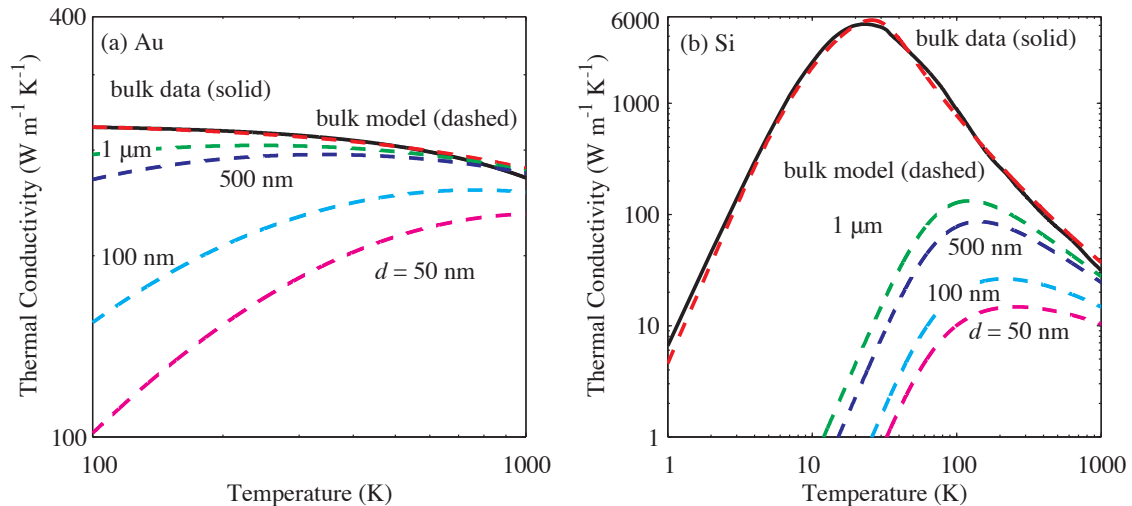

Fig. 6. (a) Electron thermal conductivity of Au as a function of temperature for bulk Au and for Au nanosystems of various limiting sizes indicated in the plot. The bulk model predictions, calculated via Eq. 95, are compared to the experimental data in Fig. 1. For these calculations, $A_{\text {ee }}=2.4 \times 10^{7} \mathrm{~K}^{-2} \mathrm{~s}^{-1}$ and $B_{\mathrm{ep}}=1.23 \times 10^{11} \mathrm{~K}^{-1} \mathrm{~s}^{-1}$ were assumed, in excellent agreement with literature values (Ivanov \& Zhigilei, 2003). Additional thermophysical parameters used for this calculation are listed in the caption of Fig. 5. The various Au nanosystem thermal conductivity is calculated via Eq. 96. (b) Phonon thermal conductivity of Si as a function of temperature for bulk Si and for Si nanoysstems of various limiting sizes in indicated in the plots. The bulk model predictions, calculated via Eq. 98, are compared to the experimental data in Fig. 1. For these calculations, the scattering coefficients were $A=1.23 \times 10^{-19} \mathrm{~s} \mathrm{~K}^{-1}, B=140 \mathrm{~K}$, and $C=1.32 \times 10^{-45} \mathrm{~s}^{3}$. In addition, the group velocity of $\mathrm{Si}$ is taken as the speed of sound, $v_{g}=8,433 \mathrm{~m} \mathrm{~s}^{-1}$, and the lattice parameter of $\mathrm{Si}$ is $a=5.430 \times 10^{-10} \mathrm{~m}$. To fit the bulk data, $d=8.0 \times 10^{-3} \mathrm{~m}$. To examine the effects of nanostructuring, $d$ is varied as indicated in the plot.

$B$ were iterated to match the data after the maximum and $C$ was taken from the literature (Mingo, 2003). The boundary scattering constant, $d$, is used as a fitting parameter to match the data at temperatures lower than the maximum. The resulting coefficients were in excellent agreement with the literature values for bulk Si (Mingo, 2003). Note that the model using Eq. 98 fits the data and captures the temperature trends extremely well showing the power of modeling the bulk phonon thermal conductivity from a fundamental energy carrier level. To examine the effects of nanostructuring on the phonon thermal conductivity, $d$ is varied to dimensions indicated in Fig. 6b. Nanostructruing greatly reduces the phonon thermal conductivity, especially at low temperatures where phonon mean free paths are long.

\section{Summary}

Modern devices, with feature sizes on the length scale of electron and phonon mean free paths, require thermal analyses different from that of the phenomenological Fourier Law. This is due to the fact that the scattering of electrons and phonons in such systems occurs predominantly at interfaces, inclusions, grain boundaries, etc., rather than within the materials comprising the device themselves. Here, electrons and phonons have been described in terms of their respective dispersion diagrams, calculated via the Schrördinger equation for electrons and atomic equations of motion for phonons. Using this information 
and density of states expressions, energy storage properties, i.e., internal energy and heat capacity, have been formulated. Lastly, applying the Kinetic Theory of Gases, the thermal conductivity expressions for metals and semiconductors have been derived. It has been shown that limiting feature sizes can result in a significant reduction in thermal conductivity. This, then, once again reinforces the idea that thermal transport on the nanoscale requires an altogether different approach from that at the macroscale.

\section{Acknowledgements}

The authors would like to acknowledge Professor Pamela M. Norris at the University of Virginia for helpful advice and for recommending the writing of this book chapter. P.E.H. would like to thank Dr. Leslie M. Phinney at Sandia National Laboratories for guidance and support. P.E.H. is appreciative for funding from the LDRD program office through the Sandia National Laboratories Harry S. Truman Fellowship Program. J.C.D. is appreciative for funding from the National Science Foundation Graduate Research Fellowship Program. Sandia is a multiprogram laboratory operated by Sandia Corporation, a wholly owned subsidiary of Lockheed Martin Corporation, for the United States Department of Energy's National Nuclear Security Administration under Contract DE-AC04-94AL85000.

\section{References}

Cahill, D. G., Ford, W. K., Goodson, K. E., Mahan, G. D., Majumdar, A., Maris, H. J., Merlin, R. \& Phillpot, S. R. (2003). Nanoscale thermal transport, Journal of Applied Physics 93(2): 793-818.

Chen, G. (2005). Nanoscale Energy Transport and Conversion: A Parallel Treatment of Electrons, Molecules, Phonons, and Photons, Oxford University Press, New York, New York.

Dove, M. T. (1993). Introduction to Lattice Dynamics, number 4 in Cambridge Topics in Mineral Physics and Chemistry, Cambridge University Press, Cambridge, England.

Griffiths, D. (2000). Introduction to Quantum Mechanics, 2nd edn, Prentice Hall, Upper Saddle River, New Jersey.

Ho, C. Y., Powell, R. W. \& Liley, P. E. (1972). Thermal conductivity of the elements, Journal of Physical and Chemical Reference Data 1(2): 279-421.

Hopkins, P. E., Norris, P. M., Phinney, L. M., Policastro, S. A. \& Kelly, R. G. (2008). Thermal conductivity in nanoporous gold films during electron-phonon nonequilibrium, Journal of Nanomaterials (418050).

Ivanov, D. S. \& Zhigilei, L. V. (2003). Combined atomistic-continuum modeling of short-pulse laser melting and disintegration, Physical Review B 68: 064114.

Kittel, C. (2005). Introduction to Solid State Physics, 8th edn, Wiley, Hoboken, New Jersey.

Mingo, N. (2003). Calculation of Si nanowire thermal conductivity using complete phonon dispersion relations, Physical Review B 68(11): 113308.

Schrödinger, E. (1926). Quantisation as a problem of characteristic values, Annalan der Physik 79: 361-376, 489-527.

Srivastava, G. P. (1990). The Physics of Phonons, Adam Hilger, Bristol, England.

Tien, C.-L., Majumdar, A. \& Gerner, F. M. (1998). Microscale Energy Transport, Taylor and Francis, Washington, D.C.

Vincenti, W. G. \& Kruger, C. H. (2002). Introduction to Physical Gas Dynamics, Krieger Publishing Company, Malabar, Florida.

Wolf, E. L. (2006). Nanophysics and Nanotechnology: An Introduction to Modern Concepts in 
Nanoscience, 2nd edn, WILEY-VCH Verlag GmbH \& Co. KGaA, Weinheim, Germany. Ziman, J. M. (1972). Principles of the Theory of Solids, 2nd edn, Cambridge University Press, Cambridge, England. 


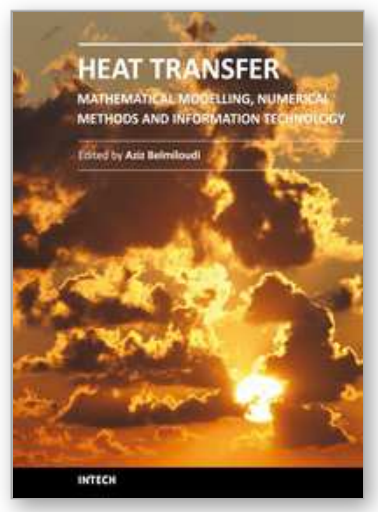

\section{Heat Transfer - Mathematical Modelling, Numerical Methods and Information Technology}

Edited by Prof. Aziz Belmiloudi

ISBN 978-953-307-550-1

Hard cover, 642 pages

Publisher InTech

Published online 14, February, 2011

Published in print edition February, 2011

Over the past few decades there has been a prolific increase in research and development in area of heat transfer, heat exchangers and their associated technologies. This book is a collection of current research in the above mentioned areas and describes modelling, numerical methods, simulation and information technology with modern ideas and methods to analyse and enhance heat transfer for single and multiphase systems. The topics considered include various basic concepts of heat transfer, the fundamental modes of heat transfer (namely conduction, convection and radiation), thermophysical properties, computational methodologies, control, stabilization and optimization problems, condensation, boiling and freezing, with many real-world problems and important modern applications. The book is divided in four sections : "Inverse, Stabilization and Optimization Problems", "Numerical Methods and Calculations", "Heat Transfer in Mini/Micro Systems", "Energy Transfer and Solid Materials", and each section discusses various issues, methods and applications in accordance with the subjects. The combination of fundamental approach with many important practical applications of current interest will make this book of interest to researchers, scientists, engineers and graduate students in many disciplines, who make use of mathematical modelling, inverse problems, implementation of recently developed numerical methods in this multidisciplinary field as well as to experimental and theoretical researchers in the field of heat and mass transfer.

\section{How to reference}

In order to correctly reference this scholarly work, feel free to copy and paste the following:

Patrick E. Hopkins and John C. Duda (2011). Introduction to Nanoscale Thermal Conduction, Heat Transfer Mathematical Modelling, Numerical Methods and Information Technology, Prof. Aziz Belmiloudi (Ed.), ISBN: 978-953-307-550-1, InTech, Available from: http://www.intechopen.com/books/heat-transfer-mathematicalmodelling-numerical-methods-and-information-technology/introduction-to-nanoscale-thermal-conduction

\section{INTECH}

open science | open minds

\section{InTech Europe}

University Campus STeP Ri

Slavka Krautzeka 83/A

51000 Rijeka, Croatia

Phone: +385 (51) 770447

Fax: +385 (51) 686166

\section{InTech China}

Unit 405, Office Block, Hotel Equatorial Shanghai

No.65, Yan An Road (West), Shanghai, 200040, China 中国上海市延安西路65号上海国际贵都大饭店办公楼 405 单元

Phone: +86-21-62489820

Fax: +86-21-62489821 
www.intechopen.com 
(C) 2011 The Author(s). Licensee IntechOpen. This chapter is distributed under the terms of the Creative Commons Attribution-NonCommercialShareAlike-3.0 License, which permits use, distribution and reproduction for non-commercial purposes, provided the original is properly cited and derivative works building on this content are distributed under the same license. 